\title{
Polymorphisme de l' $\alpha$ amylase chez le pêcher. Étude génétique
}

\author{
R Monet, B Gibault \\ INRA, station d'arboriculture fruitière, centre de recherches de Bordeaux, 33140 Pont-de-la-Maye, France
}

(Reçu le 16 octobre 1990; accepté le 13 mars 1991)

\begin{abstract}
Résumé - L' $\alpha$ amylase a été étudiée chez le pêcher par électrophorèse. Cette enzyme présente chez cette espèce 2 isozymes : l'une à migration lente AmyL, l'autre à migration rapide AmyR. La forme lente est largement répandue chez les cultivars d'origine tempérée alors que la forme rapide est rare et semble caractéristique des cultivars subtropicaux. L'étude génétique de ce système enzymatique a été entreprise; chacune de ces isozymes correspond aux produits de 2 allèles codominants d'un même gène. II existe une liaison de 4 centimorgans environ entre le locus de l' $\alpha$ amylase et celui responsable de la forme des fleurs; en revanche le locus de l' $\alpha$ amylase est indépendant de celui de la couleur de la chair du fruit.
\end{abstract}

Prunus persica $=$ pêcher $/$ électrophorèse $/$ isozymes $/$ groupe de liaison

Summary $-\alpha$-Amylase polymorphism in peach: a genetic study. $\alpha$-Amylase has been studied by electrophoresis in the peach tree. This enzyme presents 2 isozymes (fig 1); one with a slow migration, AmyL, the other with a fast migration, Amy'. The slow migrating isozyme is widespread among temperate cultivars, the fast one is scarce and seems characteristic of subtropical cultivars (table I). A genetic study of this enzymatic system has been undertaken. These isozymes are coded by 2 codominant alleles of the same locus (table II). There is a linkage of approximately 4 centimorgans between the locus of $\alpha$-amylase and that which determines the type of flower (fig 2; table III), the locus of $\alpha$-amylase is independent of that which controls the color of the flesh (table IV). Finally, the loci which determined the type of flower and the color of the flesh are also independent (table V).

Prunus persica $=$ peach tree $/$ electrophoresis $/$ isozyme $/$ linkage

\section{INTRODUCTION}

Les études du polymorphisme enzymatique sont récentes chez le pêcher. Durham et al (1985) ont signalé un polymorphisme des peroxydases, de la malate déshydrogénase et de la $D$ galacturonase. Arulsekar et al (1986) ont étudié d'une façon plus approfondie le polymorphisme de la malate déshydrogénase; l'un des locus, qui code pour cette enzyme, possède 2 allèles codominants. Messeguer et al (1987) ont mis en évidence le polymorphisme de 4 systèmes enzymatiques : l'estérase, l'isocitrate déshydrogénase, la malate déshydrogénase et la phosphatase acide. Durham et al (1987) enfin ont analysé le polymorphisme de la diaphorase, de la malate déshydrogénase et de la peroxydase et montré que les loci de ces 3 enzymes ségrégeaient de manière indépendante.

Ces études ont été faites à partir d'extraits foliaires de cultivars ou de semis de pêchers analysés en électrophorèse horizontale sur gel d'amidon.

Nous avons signalé (Reynders et Monet, 1986) un polymorphisme de l' $\alpha$ amylase en utilisant des extraits foliaires de certains de nos géniteurs. Ces extraits étaient analysés en électrophorèse horizontale sur gel de polyacrylamide.

Nous nous proposons ici d'établir la position d'une centaine de cultivars vis-à-vis de ce polymorphisme; nous étudierons ensuite son mode d'hérédité et la liaison ou l'indépendance génétique entre le locus responsable de la synthèse de l' $\alpha$ amylase et celui de 2 autres caractères à hérédité mendélienne chez le pêcher. 


\section{MATÉRIEL ET MÉTHODES}

Les 105 cultivars qui ont été utilisés pour l'étude du polymorphisme de l' $\alpha$ amylase appartenaient à la collection de la station de recherches fruitières de Bordeaux. Ils étaient âgés de 8 ans et greffés sur le porte-greffe franc GF 305.

Pour l'étude génétique nous avons utilisé des arbres de semis âgés de 4 ans obtenus par autofécondation des cultivars Redhaven (25 arbres), Weeping Flower Tree (12 arbres), Springcrest (16 arbres) et par hybridation entre le cultivar Early Sungrand et le géniteur $S(1161: 12 \times 2678$ :47)1:35 (78 arbres); le choix de cette dernière famille sera justifié plus loin.

Les protéines ont été extraites à partir de jeunes feuilles prélevées au mois de juin sur chaque cultivar ou arbre de semis étudié (l'activité $\alpha$ amylasique reste cependant très forte jusqu'à la chute des feuilles). Peu après la récolte des échantillons, les feuilles sont lyophilisées et conservées sous vide à $-30^{\circ} \mathrm{C}$.

L'électrophorèse sur gel de polyacrylamide donnant, avec des extraits bruts, de mauvais zymogrammes, nous avons été amenés à adopter une méthode d'extraction plus complexe que celle utilisée en général pour les gels d'amidon. Ainsi, pour chaque échantillon, nous procédons de la manière suivante:

- $150 \mathrm{mg}$ de feuilles lyophilisées sont broyées dans un mortier avec $50 \mathrm{mg}$ de Polyvinylpyrolidone. La poudre ainsi obtenue est versée dans $1 \mathrm{ml}$ de tampon d'extraction $\left(0,1\right.$ mol..$^{-1}$ de $L(+)$ Histidine, 5 mmol..$^{-1}$ de 1-4 Dithiothréitol, $15 \%$ de Saccharose, le pH est ajusté à 6,0 par $\mathrm{HCl}$ );

- le mélange ainsi obtenu est centrifugé pendant 20 min à $14000 \mathrm{~g}$; après centrifugation, on recueille $700 \mu \mathrm{l}$ de surnageant à l'aide d'une micropipette et le culot est éliminé;

- les protéines dissoutes dans le surnageant sont précipitées par l'addition d'une quantité égale de solution saturée de sulfate d'ammonium (ce qui correspond à une précipitation par une solution de ce sel à $50 \%$ de sa saturation). Nous laissons la précipitation se produire pendant $15 \mathrm{~min}$;

- les protéines précipitées sont récupérées par une nouvelles centrifugation de $15 \mathrm{~min}$ à $14000 \mathrm{~g}$. Le tampon est éliminé, les protéines restent dans le culot;
- pour les besoins de l'électrophorèse, il faut débarrasser les protéines du sulfate d'ammonium. Le dessalage se fait de la manière suivante : les protéines sont redissoutes dans $500 \mu \mathrm{l}$ de tampon d'extraction et passées sur colonne IBF 11 contenant $4 \mathrm{ml}$ de gel Trisacryl GF 05. L'élution se fait toujours avec le tampon d'extraction; la fraction de l'éluat contenant les protéines passe la première. Elle est recueillie et sera utilisée pour l'électrophorèse. (Toutes les opérations décrites ci-dessus se font à la température de $4^{\circ} \mathrm{C}$ ). La conservation de ces extraits à $-30^{\circ} \mathrm{C}$ prolonge leur activité enzymatique pendant plusieurs mois.

Nous utilisons pour l'électrophorèse des gels à $7,5 \%$ d'acrylamide. Ces gels, de $2 \mathrm{~mm}$ d'épaisseur, de $26 \mathrm{~cm}$ de largeur et $12,5 \mathrm{~cm}$ de longueur permettent l'électrophorèse simultanée de 20 échantillons. Le tampon d'électrode (utilisé aussi dans la confection du gel) a la composition suivante : Tris $\mathrm{HCl} 0,15 \mathrm{~mol}^{-1}$, Glycine $0,15 \mathrm{~mol}^{-1}{ }^{-1}, \mathrm{pH}$ 8. L'électrophorèse est horizontale, la tension appliquée au gel est de $16 \mathrm{~V}$ par $\mathrm{cm}$.

Après migration, les gels sont placés en incubation pendant $1 \mathrm{~h}$ à $30^{\circ} \mathrm{C}$ dans une solution d'amidon (amidon soluble $1 \%$ dans tampon acétate à $\mathrm{pH} 5,6$ ). La révélation de l' $\alpha$ amylase se fait dans une solution d'iode-iodure de potassium (I $0,01 \mathrm{~mol}^{-1}{ }^{-1}$, IK 0,03 mol. $1^{-1}$ ).

\section{RÉSULTATS}

\section{Polymorphisme de l' $\alpha$ amylase, répartition des cultivars}

Trois phénotypes (fig 1) sont reconnaissables concernant l' $\alpha$ amylase, les cultivars qui possèdent une bande à migration lente, les cultivars qui possèdent une bande à migration rapide, enfin les cultivars qui possèdent à la fois la bande lente et la bande rapide.

Le tableau I donne la répartition de 105 cultivars concernant ce polymorphisme. II faut noter ceci :
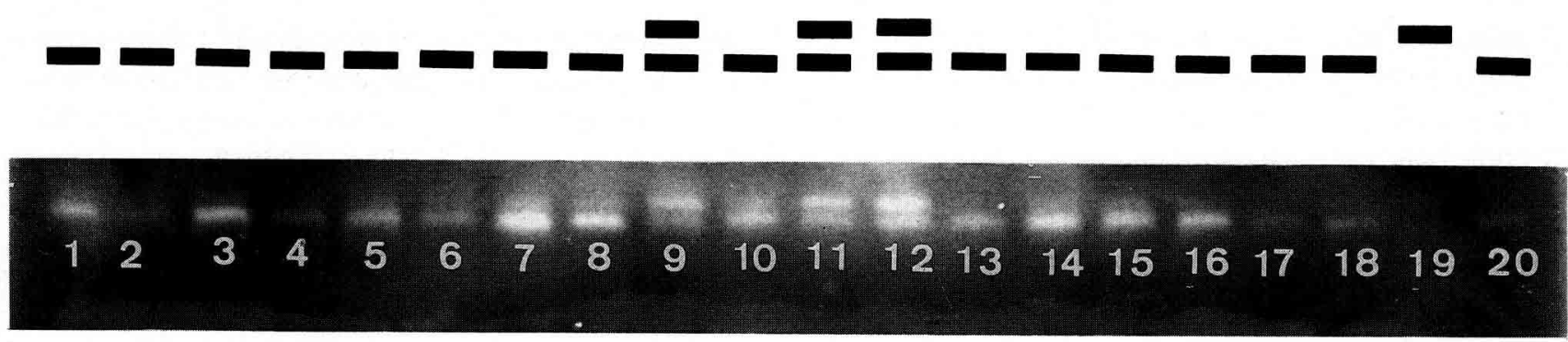

Fig 1. Zymogramme de l' $\alpha$ amylase de 20 cultivars: Merrill Early O Henry (1), Nectared 6 (2), Springtime (3), Orlandine (4), Armking (5), Harko (6), Redtop (7), Fuzalode (8), Robin (9), Babygold 6 (10), Redwing (11), Springcrest (12), Nerine (13), Troubador (14), July Lady (15), Redcal (16), Jing Hong (17), Snowqueen (18), Bailfeng (19), Redhaven (20). Au-dessus, en traits noirs, représentation schématique du zymogramme. 
Tableau I. Répartition de 105 cultivars de pêcher selon le polymorphisme de l' $\alpha$ amylase.

Cultivars à bande lente :

Ambergold, Amsden, Andross, Armking, Babygold 5, Babygold 6, Babygold 7, Babygold 8, Barbara, Calred, Croce del Sur, Desert gold, Dixired, Earlyglo, Early Redhaven, Early Sungrand, Elegant Lady, Everts, Fairhaven, Fantasia, Firered, Flamecrest, Flavorcrest, Fuzalode, Garnet Beauty, Glohaven, Golo, Hardired, Harken, Harko, Henry Moulin, Independence, JH Hale, July Lady, Jing Hong, Kakamas, Klant, La Red, la Gold, Loadel, Loring, Maybelle, Merriam, Merricle, Merrill Angelus, Merrill Early O Henry, Merrill Gemfree, Merrill O Henry, Michelini, Morton, Nectared 6, Nerine, Niagara, Norman, Orlandine, Peento Late, Peento Red, Peento Yellow, Philp, Primerose, Redcal, Redhaven, Redjune, Redtop, Royal April, Royal Gold, Royal May, Rubidoux, Shasta, Silver Lode, Snowqueen, Southland, Springold, Springred, Springtime, Suncrest, Sundanell, Summergrand, Summertime, Sweeny, Troubador, Tuft, Vesuvio, Vivian, Walgrant.

Cultivars à 2 bandes :

Cashemere, Diamante, Early Belle, Early Grande, Flordaking, Flordaprince, Honey, Magno, Orion, Peking, Redskin, Redwing, Robin, Shanghaï, Silver Logan, Sprincrest.

Cultivars à bande rapide :

Baîfeng, Kiang Si, Maravilla, Weeping flower Tree.

- tous les cultivars (à une exception près : Croce del Sur) qui ont une $\alpha$ amylase à migration lente sont de climat tempéré;

- les cultivars à 2 bandes, proviennent en majorité de régions subtropicales; les exceptions concernent Redwing, Robin, Springcrest et Redskin. Redwing et Robin ont un ancêtre commun Peento d'origine subtropicale; Springcrest a comme parent Robin; enfin Redskin est un hybride entre $\mathrm{JH}$ Hale (cultivar à bande lente) et Elberta issu lui-même de Chinese Cling originaire de la région de Shanghaï;

- les cultivars à bande rapide, rares dans notre étude, sont tous des cultivars de climat subtropical.

\section{Déterminisme génétique de l' $\alpha$ amylase chez le pêcher}

L'autofécondation d'un cultivar à bande lente tel que Redhaven donne «enfin l'autofécondation d'un cultivar à 2 bandes tel que Springcrest donne une famille avec des individus à bande lente, à bande rapide et à 2 bandes (tableau II). L'écart que l'on constate ici par rapport à des proportions mendéliennes simples (1:2:1) tient au géniteur Springcrest dont les embryons immatures présentent une mortalité élevée à la germination.

On peut considérer que les 2 isozymes de l' $\alpha$ amylase sont les produits de l'expression de 2 allèles codominants d'un même locus. Nous appelerons AmyL l'allèle qui détermine l' $\alpha$ amylase lente et AmyR l'allèle qui détermine l' $\alpha$ amylase rapide.

\section{Étude des liaisons génétiques entre le locus de l' $\alpha$ amylase et ceux qui déterminent le type de fleur et la couleur de la chair du fruit}

Nous avons utilisé pour cette étude une famille de 78 arbres, qui résulte du croisement entre la nectarine Early Sungrand et le géniteur $S$ $(1161: 12 \times 2678: 47) 1: 35$. Ce dernier provient de la génération $F_{2}$ obtenue en croisant d'abord un géniteur ayant une $\alpha$ amylase à migration lente

Tableau II. Répartition des phénotypes pour l' $\alpha$ amylase dans les descendances par auto-fécondation de Redhaven, Weeping flower et Springcrest.

\begin{tabular}{lllll}
$\begin{array}{l}\text { Cultivar } \\
\text { autofécondé }\end{array}$ & $\begin{array}{l}\text { Individus à } \\
\text { bande lente }\end{array}$ & $\begin{array}{l}\text { Individus à } \\
\text { bande rapide }\end{array}$ & $\begin{array}{l}\text { Individus à } \\
2 \text { bandes }\end{array}$ & Total \\
\hline
\end{tabular}

$\begin{array}{lrrrr}\text { Redhaven } & 25 & 0 & 0 & 25 \\ \text { Weeping flower } & 0 & 12 & 0 & 12 \\ \text { Springcrest } & 3 & 2 & 11 & 16\end{array}$


S1161:12 par un géniteur ayant une $\alpha$ amylase à migration rapide $\mathrm{S} 2678: 47$ et en autofécondant ces hybrides $F_{1}$.

Le croisement Early Sungrand par $\mathrm{S}(1161: 12$ $x$ 2678:47)1:35 constitue un test cross pour les caractères suivants : la forme de la fleur, la couleur de la chair du fruit et l' $\alpha$ amylase. En effet le géniteur Early Sungrand est homozygote récessif pour les 2 premiers (fleur rosacée : sh sh et chair jaune : $y$ y et homozygote codominant pour le $3^{e}$ ( $\alpha$ amylase lente Amy $L$ AmyL). Le géniteur $S(1161: 12 \times 2678: 47) 1: 35$, au contraire, s'est révélé, a posteriori, à l'observation de la famille issue du croisement, hétérozygote pour ces 3 caractères.

Ce test cross va permettre ainsi une étude des liaisons génétiques entre ces différents caractères.

\section{Étude de la liaison génétique existant entre le locus déterminant le type d' $\alpha$ amylase et celui déterminant le type de fleur}

Rappelons qu'il existe chez le pêcher 2 types de fleurs à déterminisme monogénique : le phénotype fleur "Campanulée" présent chez le parent $S(1161: 12 \times 2678: 47) 1: 35$, ce caractère est dominant et peut correspondre aux génotypes $S h$ Sh ou Sh sh; le phénotype fleur "Rosacée» récessif, de génotype sh sh. La famille issue du croisement S(1161:12 x 2678:47)1:35 avec Early Sungrand donne des individus à fleur rosacée et à fleur campanulée et des individus à 2 bandes ou à 1 bande lente en ce qui concerne l' $\alpha$ amylase (fig 2). C'est donc que le géniteur $\mathbf{S}(1161: 12$ $x$ 2678:47)1:35 était hétérozygote pour ces caractères. La répartition des individus concernant les associations 2 à 2 de ces phénotypes est donnée dans le tableau III.

L'examen des fréquences marginales de ce tableau montre que la répartition des phénotypes fleur campanulée/fleur rosacée, $\alpha$ amylase
Tableau III. Répartition des phénotypes dans la descendance du croisement S(1161:12 x 2678:47)1:35 x Early Sungrand pour les caractères : type de fleur et $\alpha$ amylase.

Phénotypes $\alpha$ amylase $\alpha$ amylase Total 2 bandes 1 bande lente

Fleur campanulée Fleur rosacée $1(19,5) \quad 36(19,5) \quad 37(39)$

Total $39(19,5) \quad 2(19,5) \quad 41(39)$ $40(39) \quad 38(39) \quad 78$

Les chiffres entre parenthèses sont les valeurs théoriques pour une disjonction 1:1 (fréquences marginales) et 1:1:1:1 (fréquences des caractères associés 2 à $2, \chi^{2}$ calculé $=62,9$ $\chi^{2} 0,05=7,81$ pour 3 degrés de liberté).

2 bandes/ $\alpha$ amylase 1 bande n'est pas significativement différente des proportions 1:1. Quant à la répartition des 4 phénotypes, combinaison 2 à 2 des précédents, elle est significativement différente des proportions théoriques 1:1:1:1 (chiffres entre parenthèses) caractéristique, dans le cas d'un test cross, de l'indépendance génétique. II y a, en effet un fort déficit des phénotypes : fleur campanulée / $\alpha$ amylase 2 bandes, fleur rosacée/ $\alpha$ amylase 1 bande lente alors que les phénotypes fleur campanulée/ $\alpha$ amylase 1 bande lente, fleur rosacée/ $\alpha$ amylase 2 bandes sont excédentaires.

Nous sommes donc en présence d'une forte liaison entre les loci déterminant la forme de la fleur et ceux qui déterminent le type d' $\alpha$ amylase. L'intensité de la liaison est mesurée par le pourcentage des phénotypes recombinés soit :

$$
(2+1 / 78) \times 100=3,8 \%
$$

soit environ 4 centimorgans.

Early Sungrand étant à fleur rosacée et à $\alpha$ amylase à migration lente (génotype sh sh, Amyl
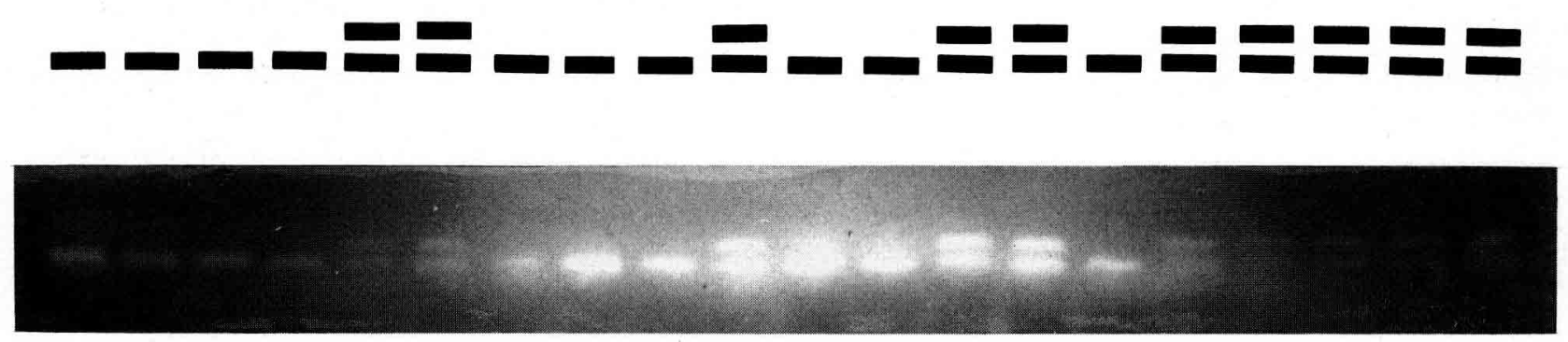

Fig 2. Zymogramme de l' $\alpha$ amylase : de gauche à droite : arbres 47 à 66 de la famille $S(1161: 12 \times 2678: 47) 1: 35 \times$ Early Sungrand. Au-dessus, en traits noirs, représentation schématique du zymogramme. 
AmyL), le géniteur $S(1161: 12 \times 2678: 47) 1: 35$ doit être nécessairement :

$$
\text { Sh AmyL }
$$

Ce qui donne comme gamètes parentaux : Sh, Amyl et sh, Amyr.

\section{Indépendance des loci déterminant la couleur de la chair du fruit et l' $\alpha$ amylase}

Chez le pêcher, la couleur de la chair du fruit est à déterminisme mendélien. Le caractère chair blanche dominant peut correspondre à 2 génotypes $Y Y$ et $Y y$; le phénotype chair jaune est récessif, il correspond au génotype yy. La famille issue du croisement $S(1161: 12 \times 2678: 47) 1: 35$ par Early Sungrand donne des individus dont les fruits sont à chair blanche ou jaune; c'est donc que le géniteur $S(1161: 12 \times 2678: 47) 1: 35$ était hétérozygote pour le caractère couleur de la chair (Early Sungrand étant yy). Le tableau IV donne la répartition des phénotypes concernant les associations 2 à 2 de la couleur de la chair et de l' $\alpha$ amylase. Les fréquences marginales font apparaître un léger déficit de "chairs blanches" (et donc un excès de "chairs jaunes") par rapport aux fréquences théoriques d'une disjonction mendélienne alors que pour l' $\alpha$ amylase, fréquences théoriques et fréquences observées sont très proches. Si l'on s'intéresse aux 4 combinaisons génotypiques possibles de ces caractères pris 2 à 2 , les fréquences observées ne sont pas ici statistiquement différentes des proportions théoriques 1:1:1:1 caractéristiques, dans le cas d'un test cross, de l'indépendance génétique des 2 couples d'allèles $Y y$ et AmyL AmyR.

Tableau IV. Répartition des phénotypes dans la descendance du croisement $S(1161: 12 \times 2678: 47) 1: 35 \times$ Early Sungrand pour les caractères : couleur de la chair du fruit et $\alpha$ amylase.

\begin{tabular}{llll} 
Phénotypes & $\begin{array}{l}\alpha \text { amylase } \alpha \text { amylase } \\
2 \text { bandes } 1 \text { bande lente }\end{array}$ & Total \\
\hline Chair blanche & $19(19,5)$ & $15(19,5)$ & $34(39)$ \\
Chair jaune & $21(19,5)$ & $23(19,5)$ & $44(39)$ \\
Total & $40(39)$ & $38(39)$ & 78
\end{tabular}

Les chiffres entre parenthèses sont les valeurs théoriques pour une disjonction 1:1 (fréquences marginales) et 1:1:1:1 (fréquences des caractères associés 2 à $2, \chi^{2}$ calculé $=1,79$ $\chi^{2} 0,05=7,81$ pour 3 degrés de liberté).

\section{Indépendance des loci déterminant le type de fleur et la couleur de la chair}

Nous avions déjà établi cette indépendance génétique (Monet et Bastard, 1983). Les fréquences observées dans cette nouvelle étude (tableau V) confirment encore cette situation puisqu'elles ne sont pas statistiquement différentes des proportions théoriques 1:1:1:1.

\section{DISCUSSION ET CONCLUSION}

L'étude du polymorphisme de l' $\alpha$ amylase chez le pêcher semble montrer que la répartition des cultivars en fonction du type d'isoenzyme qu'il possède n'est pas une répartition aléatoire mais coïncide avec les 2 groupes adaptatifs que l'on distingue habituellement chez cette espèce. Les variétés adaptées aux climats tempérés possèdent de façon préférentielle l' $\alpha$ amylase à migration lente, les variétés adaptées à des climats subtropicaux possèdent généralement une $\alpha$ amylase à migration rapide ou les 2 isoamylases. Les cultivars subtropicaux sont souvent des hybrides entre un cultivar subtropical d'origine locale et un cultivar tempéré qui apporte les caractères technologiques du fruit; leur état hétérozygote pour l' $\alpha$ amylase n'est donc pas en contradiction avec la situation qui est décrite.

Cette répartition s'explique-t-elle par un avantage sélectif de chacune de ces isoenzymes dans chaque type de climat comme cela semble être le cas pour d'autres systèmes enzymatiques (Alahiotis, 1982) ou bien s'agit-il d'une explication tout autre (phénomène de convergence, effet fondateur )? Seule l'étude d'un plus

Tableau V. Répartition des phénotypes dans la descendance du croisement S(1161:12 x 2678:47)1:35 x Early Sungrand pour les caractères : type de fleur et couleur de la chair du fruit.

\begin{tabular}{llll} 
Phénotypes & $\begin{array}{c}\text { Fleur } \\
\text { campanulée }\end{array}$ & $\begin{array}{c}\text { Fleur } \\
\text { rosacée }\end{array}$ & Total \\
\hline Chair blanche & $15(19,5)$ & $19(19,5)$ & $34(39)$ \\
Chair jaune & $22(19,5)$ & $22(19,5)$ & $44(39)$ \\
Total & $37(39)$ & $41(39)$ & 78
\end{tabular}

Les chiffres entre parenthèses sont les valeurs théoriques pour une disjonction 1:1 (fréquences marginales) et 1:1:1:1 (fréquences des caractères associés 2 à $2, \chi^{2}$ calculé $=1,69$ $\chi^{2}{ }_{0,05}=7,81$ pour 3 degrés de liberté). 
grand nombre de cultivars subtropicaux pourrait permettre de conclure. Quoiqu'il en soit cette répartition pose question.

La liaison entre l' $\alpha$ amylase et le type de fleur est la premiere liaison mise en évidence entre un marqueur biochimique et un caractère morphologique chez le pêcher.

La netteté et la simplicité d'interprétation des zymogrammes font de l' $\alpha$ amylase un excellent marqueur biochimique du pêcher. Au même titre que les marqueurs morphologiques à hérédité mendélienne déjà répertoriés il va contribuer à l'amélioration de la connaissance génétique de l'espèce.

\section{RÉFÉRENCES}

Alahiotis SN (1982) Adaptation of Drosophila enzymes to temperatures. IV Natural selection at the alcohol dehydrogenase locus. Genetica 59, 81-87
Arulsekar S, Parfitt DE, Beres W, Hansche PE (1986) Genetics of malate dehydrogenase isozymes in the peach. J Hered 77, 49-51

Durham RE, Moore GA, Sherman WB (1985) Isozyme banding patterns and inheritance in peach. Hort science 20, 543 (abstract 160)

Durham RE, Moore GA, Sherman WB (1987) Isozyme banding patterns and their usefulness as genetic markers in peach. J Am Soc Hortic Sci 112, 10131018

Messeguer R, Arus P, Carrera M (1987) Identification of peach cultivars whith pollen isozymes. Sci Hortic 31, 107-117

Monet R, Bastard Y (1983) Nouveaux cas de ségrégation indépendante de caractères mendéliens chez le pêcher. Agronomie 3, 387-390

Reynders S, Monet R (1986) Évolution, au cours du temps, de la consanguinité des variétés de pêcher. Étude des distances génétiques entre quelques géniteurs. In : $6^{\theta}$ Colloque des recherches fruitières. INRA, CTIFL, 29-41 